\title{
Hyperspectral Imaging for Differentiating Glyphosate-Resistant and Glyphosate-Susceptible Italian Ryegrass
}

\author{
Yanbo Huang ${ }^{1 *}$, Matthew A. Lee ${ }^{2}$, Vijay K. Nandula ${ }^{1}$, Krishna N. Reddy ${ }^{1}$ \\ ${ }^{1}$ United States Department of Agriculture, Agricultural Research Service, Stoneville, MS, USA \\ ${ }^{2}$ Electrical and Computer Engineering, Mississippi State University, Mississippi State, MS, USA \\ Email: *yanbo.huang@ars.usda.gov
}

How to cite this paper: Huang, Y., Lee, M.A., Nandula, V.K. and Reddy, K.N. (2018) Hyperspectral Imaging for Differentiating Glyphosate-Resistant and Glyphosate-Susceptible Italian Ryegrass. American Journal of Plant Sciences, 9, 1467-1477. https://doi.org/10.4236/ajps.2018.97107

Received: April 17, 2018

Accepted: June 18, 2018

Published: June 21, 2018

Copyright $\odot 2018$ by authors and Scientific Research Publishing Inc. This work is licensed under the Creative Commons Attribution International License (CC BY 4.0).

http://creativecommons.org/licenses/by/4.0/

\begin{abstract}
Glyphosate is widely used in row crop weed control programs of glyphosate-resistant (GR) crops. With the accumulation of glyphosate use, several weeds have evolved resistance to glyphosate. In order to control GR weeds for profitable crop production, it is critical to first identify them in crop fields. Conventional method for identifying GR weeds is destructive, tedious and labor-intensive. This study developed hyperspectral imaging for rapid sensing of Italian ryegrass (Lolium perenne ssp. multiflorum) plants to determine if each plant is GR or glyphosate-susceptible (GS). In image analysis, a set of sensitive spectral bands was determined using a forward selection algorithm by optimizing the area under the receiver operating characteristic between GR and GS plants. Then, the dimensionality of selected bands was reduced using linear discriminant analysis. At the end the maximum likelihood classification was conducted for plant sample differentiation of GR Italian ryegrass from GS ones. The results indicated that the overall classification accuracy is between $75 \%$ and $80 \%$. Although the accuracy is lower than the classification of Palmer amaranth (Amaranthus palmeri S. Wats.) in our previous study, this study provides a rapid, non-destructive approach to differentiate between GR and GS Italian ryegrass for improved site-specific weed management.
\end{abstract}

\section{Keywords}

Hyperspectral Imaging, Glyphosate Resistance, Italian Ryegrass

\section{Introduction}

Glyphosate [N-(phosphonomethyl)-glycine] is the most widely used herbicide with its increasing use in transgenic, glyphosate-resistant (GR) cropping systems 
[1]. However, the repeated and intense use of glyphosate has exerted a high selection pressure on weed populations and has resulted in the evolution of GR weeds. To date, a total of 41 weed species have exhibited resistance to glyphosate worldwide and 9 of them have been documented in Mississippi [2].

Italian ryegrass (Lolium perenne L. ssp. Multiflorum (Lam.) Husnot) is one of the GR weeds that was first reported in Chile in 2001 in fruit and orchard farming systems, followed by Brazil, Argentina, Spain, Italy, Switzerland, New Zealand and Japan in different agronomic situations [2]. In the United States, GR Italian ryegrass was first reported in Oregon in orchards in 2004 and in Mississippi in GR cotton and GR soybean fields in 2005 [2]. Since then, Arkansas, California, North Carolina, Tennessee, and Louisiana have also reported GR Italian ryegrass consecutively until 2016 [2]. Italian ryegrass is an erect winter annual with a biennial-like growth habit. It grows vigorously in winter and early spring and is highly competitive in crop fields. GR Italian ryegrass populations could seriously jeopardize preplant burndown options and planting operations in reduced-tillage crop production systems of Mississippi. In order to effectively manage and control GR Italian ryegrass in crop fields, it is necessary to identify GR and glyphosate-susceptible (GS) Italian ryegrass and map the distribution of the GR and GS Italian ryegrass patches and clusters.

The conventional method for differentiating GR and GS plants is destructive and assesses the plant physiological and biochemical changes before and after glyphosate treatment. During such an assessment, glyphosate is treated on the whole plant, single leaves or leaf disks [3] [4]. With glyphosate treatment, GS plants will show symptoms of injury with time after treatment while the GR plants will have no or much less symptoms of injury depending on the resistance mechanism and growing conditions. This method is regularly used by weed scientists and is effective and accurate for conducting plant physiological studies on GR and GS weeds. However, this method is destructive, tedious and labor intensive.

With glyphosate treatment, plants will indicate changes in physiological, biochemical and cell structures [5]. These changes can be illustrated as plant stress to be detected by measuring and analyzing plant optical spectral characteristics. Hyperspectral plant sensing uses sensors that subdivide the electromagnetic spectrum (ultra-violet, visible, and infrared) into a large number of wavelength bins (typically over 100), which allows detection of subtle changes in plant spectra. This technology has been utilized in numerous agricultural studies, notably identification of plant stress caused by drought, nutrient deficiencies, pest infestations, and herbicide applications [6] [7] [8].

In order to develop a rapid, non-destructive method for differentiating GR and GS weeds, Reddy et al. [9] conducted a pilot study to differentiate GR Palmer amaranth (Amaranthus palmeri S. Wats.) from GS Palmer amaranth using hyperspectral imaging technology. In these experiments, three different plant sample populations were evaluated and consistently demonstrated that GS and 
GR Palmer amaranth could be directly differentiated through spectral data analysis with accuracies over $90 \%$ without glyphosate treatment.

With our success in previous research with GR and GS Palmer amaranth differentiation, we intend to further study and test if the same approach could be transferred to other GR weed species. Therefore, the objective of this research was to develop a hyperspectral imaging method for rapid, non-destructive measurement, processing and analysis of spectral data for differentiation between GR and GS Italian ryegrass.

\section{Materials and Methods}

\subsection{Experimental Plant Samples}

For this study, 226 Italian ryegrass plants were grown from seeds, transplanted ( 2 weeks after emergence) in $6 \mathrm{~cm}$ by $6 \mathrm{~cm}$ by $6 \mathrm{~cm}$ black plastic pots containing a commercial potting mix in a greenhouse at the United States Department of Agriculture, Agricultural Research Service, Crop Production Systems Research Unit at Stoneville, Mississippi. The 226 plants consisted of 119 GR and 107 GS Italian ryegrass.

\subsection{Plant Imaging}

The plants were imaged using a Resonon Pika II VNIR (Visible + Near Infrared) hyperspectral imaging system (Resonon, Bozeman, MT). The Pika II camera was mounted approximately $1 \mathrm{~m}$ above the sample stage, which (when combined with the lens magnification) resulted in sub-millimeter pixel sizes. The camera has $2.1 \mathrm{~nm}$ spectral range and 12 bit dynamic range. The spectral range of the camera is $400-900 \mathrm{~nm}$, with 240 narrow wavelength bands evenly spaced in the range. The high spatial resolution of the imaging settings ensured that a very large number of pure plant pixels were present in the images. During imaging, the sample stage was covered by black felt cloth, which has almost no spectral reflection, to provide good contrast between the plants and the background in the acquired images. The good contrast simplified segmentation of the plant pixels from the non-plant pixels in the images as well.

The plants were first imaged approximately 3 weeks after transplanting, and then 4 and 5 weeks after transplanting. At the first imaging, each plant had an average of about 7 tillers, and at the second imaging, the number of tillers were 14. At the third, the number of tillers was over 20 on average. The SpectrononPro software (Resonon, Bozeman, MT) developed for the Pika II hyperspectral imaging system combines the white reference and dark current measurements with the images of the plants to calculate spectral reflectance for each pixel of the images. Figure 1 shows the Pika II hyperspectral imaging system with the SpectrononPro interface and the example spectral curves (they are the results of Equation (4) in the next section) of the Italian ryegrass plants and black felt cloth.

\subsection{Image Processing Algorithm}

The calculated reflectance values were normalized to remove artifacts caused by 


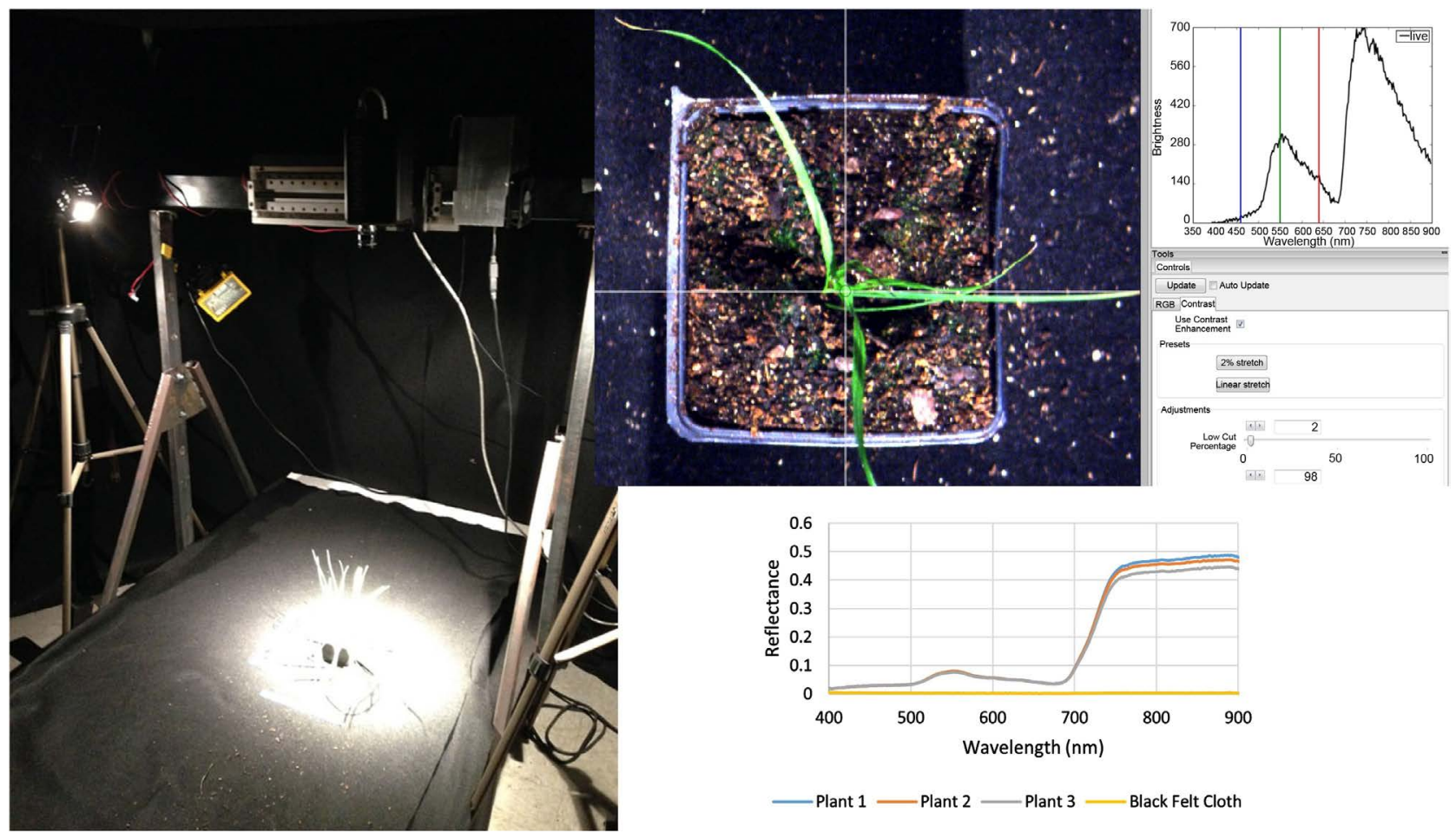

Figure 1. The Pika II hyperspectral imaging system, the SpectrononPro interface and the example spectral curves of the Italian ryegrass plants and black felt cloth.

height variation in the plants. The normalization was accomplished by dividing the reflectance of each pixel at a wavelength by its vector magnitude across all the wavelengths. The equation for the normalization is as follows:

$$
R_{i j k}=\frac{r_{i j k}}{\sqrt{\sum_{k=1}^{n} r_{i j k}^{2}}}
$$

where the reflectance $r_{i j k}$ is the original reflectance of the pixel at $(i, j)$ for the $k$ th wavelength in the total $n$ wavelengths.

After reflectance normalization, images were segmented to separate the pixels of the plants from the pixels of the background. The segmentation was conducted by assuming that the plant was the only green object in each image. To segment the green object out of each image, a three-dimensional vector was formed using the reflectance values at $450 \mathrm{~nm}$ (Blue), $550 \mathrm{~nm}$ (Green), and 650 $\mathrm{nm}$ (Red) as:

$$
v_{i j}=\left\langle r_{i j, 450 \mathrm{~nm}}, r_{i j, 550 \mathrm{~nm}}, r_{i j, 650 \mathrm{~nm}}\right\rangle
$$

The vector was then normalized similarly as:

$$
V_{i j}=\frac{v_{i j}}{\sqrt{r_{i j, 450 \mathrm{~nm}}^{2}+r_{i j, 550 \mathrm{~nm}}^{2}+r_{i j, 650 \mathrm{~nm}}^{2}}}
$$

The dot product between the normalized vector $V_{i j}$ and the unit vector $\langle 0,1,0\rangle$ was, then, thresholded to identify green pixels in the images. Through trial and error, the pixels with values over the threshold of 0.8 were found to be 
appropriate for identifying plant pixels. In using this technique, when the vector $v_{i j}$ has a small magnitude, there is risk to label dark pixels as green in addition to labeling plant pixels. Therefore, a correct program was coded and implemented to prevent dark pixels from being labeled as green pixels by labeling the pixels with the magnitude of $v_{i j}$ less than 0.2 as background.

Finally, the normalized reflectance $R_{i j k}$ values for the pixels labeled as plant were averaged to obtain the mean reflectance for the plant in the image:

$$
\bar{R}_{k}=\frac{1}{\text { Number of Plant Pixels }} \sum_{i^{*} j^{*}} R_{i j k} \quad(k=1,2, \cdots, n)
$$

where $i^{*}$ and $j^{*}$ are the locations of plant pixels in the image.

\subsection{Data Analysis}

Data analysis was conducted on the mean reflectance for the plants as shown in Equation (4). The data set was randomly divided into training (89 GR and 80 GS) and testing (30 GR and 27 GS) subsets. A classification model was calibrated with the training data set, and then tested using the testing data set. The model was calibrated using a forward selection algorithm to select characteristic wavelengths. Then, Fisher's Linear Discriminant Analysis (FLDA) was used to reduce the dimensionality of the set of selected wavelength bands. At last, the maximum likelihood classifier was used for classification between GR and GS Italian ryegrass.

\subsection{Band Selection}

Due to the large number of spectral bands and the limited number of training samples, it was necessary to use only some of the available bands to avoid diminishing results. Diminishing results are caused when there are not enough training samples to estimate joint probability distributions in high dimensional feature spaces [10]. In our previous research for differentiating GR and GS Palmer amaranth, the forward selection algorithm was conducted for spectral band selection [9]. The band selection process began with an empty set of selected bands. The bands were sorted based on the area under the receiver operating characteristic (ROC) curve [11]. The area under the ROC measures how much overlap the clusters of the GS and GR plant classes have in each wavelength band. If the means of the GS and GR classes are farther apart, or the standard deviations of one or both decrease, the area under the ROC will increase. Usually, the classification accuracy is better when features with greater areas under the ROC. The forward selection algorithm repeatedly selected the band that increased the area under the ROC the most (as computed on the set of selected bands plus the new candidate band). The band selection process did this by first computing the area under the ROC on the selected band set with each candidate band added in the order from best to worst. After the area under the ROC has been computed for all possible candidates and selections, the candidate band that had the highest score is added to the selected set. The process of adding and selecting a new band was repeated until a predetermined number of bands were 
selected.

\subsection{Dimensionality Reduction of Selected Bands}

A problem using ROC is that it can only be computed on a one dimensional feature vector. This means that when the dimensionality of the selected bands set is greater than one, all the information contained within those selected bands has to be summarized by a one dimensional vector (that is, reduce the dimensionality to one). One way to reduce the dimensionality is to compute a weighted sum of all the bands. FLDA does this by computing the weights that optimize the ratio of between class scatter divided by within class scatter [12]. Thus, the selected band set is summarized so that class separation, and therefore, area under the ROC, is optimized.

\subsection{Classification}

After the spectral bands were selected and dimensionality reduced, the training data set was used to train the maximum likelihood classifier [13]. The maximum likelihood classifier used the clusters of GS and GR plants to compute the probability of an unknown test sample belonging to one of the classes. The class with the greatest probability was labeled and selected. In order to compute the probability, the distribution of the data must be fit to a probability distribution function. There are many common distribution functions, but in this experiment the data was assumed to fit the Gaussian distribution function. Thus, the means and the variances of GS and GR plants were estimated from the training data. This information was, then, used to estimate the probability of unknown samples belonging to GS or GR classes using the Gaussian probability distribution function equation.

\subsection{Performance Evaluation}

The performance of the classifier was determined in two different ways. The first was to compute a calibration accuracy using leave-one-out validation on the training data [13]. This is considered a biased performance metric since all the samples were used to select bands and reduce dimensionality. Thus, the performance of the classifier in use is usually worse than in model calibration. The second was to use the testing data set, which was not used in band selection and dimensionality reduction, to compute the validation accuracy.

The results of both performance metrics were organized into confusion matrices. To enhance the performance evaluation, a permutation study was conducted by repeating the classifications 100 times and computing the mean and standard deviation of the confusion matrices. Since the training and testing data sets were chosen randomly, each permutation had different training and testing data sets to determine the overall performance of the process.

\section{Results and Discussion}

As mentioned above, the Italian ryegrass plants were imaged 3, 4 and 5 weeks 
after transplanting. With each time of data 15 most sensitive wavelength bands were selected (Table 1). At three weeks after transplanting, the mean overall validation accuracy of classification settled on about $75.1 \%$ with the number of selected wavelength bands as shown in Figure 2. The standard deviation of the overall accuracy remained relatively constant regardless of number of bands selected with in the $95 \%$ confidence intervals of the mean overall accuracy displayed as error bars in the figure. At four weeks after transplanting, the mean overall validation accuracy of classification settled on about $80.2 \%$ with the number of selected wavelength bands as shown in Figure 3. The error bars in Figure 3 also indicate the $95 \%$ confidence interval with a quite constant standard deviation. Confusion matrices were computed with the increase of selected bands. At five weeks after transplanting, the mean overall validation accuracy of classification settled on about $75.4 \%$ with the number of selected wavelength bands as shown in Figure 4. The error bars in the figure also indicate the 95\% confidence interval with a fairly constant standard deviation. Confusion matrices were computed with the increase of selected bands. Tables 2-4 are the final confusion matrices for GR and GS Italian ryegrass classification at three, four and five weeks after transplanting, respectively. Therefore, the results indicated that GS and GR Italian ryegrass could be differentiated the classification accuracy in the range of $75 \%-80 \%$ regardless of number of a few weeks after emergence.

Table 1. Sensitive wavelength bands selected from the images acquired at 3, 4 and 5 weeks after transplanting (WAT).

\begin{tabular}{ccccccccccccccccc}
\hline WAT & \multicolumn{11}{c}{ Wavelength Bands (nm) } \\
\hline 3 & 402.7 & 592.0 & 596.2 & 619.3 & 621.4 & 627.7 & 631.9 & 640.4 & 663.5 & 669.8 & 741.3 & 753.9 & 764.4 & 880.1 & 892.7 \\
4 & 417.4 & 507.9 & 514.2 & 577.3 & 589.9 & 608.8 & 657.2 & 739.2 & 785.5 & 793.9 & 806.5 & 819.1 & 829.6 & 835.9 & 854.9 \\
5 & 396.4 & 413.2 & 423.7 & 425.8 & 440.6 & 470.0 & 669.8 & 686.6 & 735.0 & 739.2 & 741.3 & 747.6 & 762.3 & 768.6 & 791.8 \\
\hline
\end{tabular}

Table 2. Confusion matrix at three weeks after transplanting with standard deviation in parentheses.

\begin{tabular}{|c|c|c|c|}
\hline Actual Class & GR & GS & Accuracy \\
\hline GR & $23.1(2.2)$ & $6.9(2.2)$ & $76.9 \%$ \\
\hline GS & $7.2(2.6)$ & $19.7(2.6)$ & $73.2 \%$ \\
\hline Overall Accuracy & & & $75.1 \%$ \\
\hline
\end{tabular}

Table 3. Confusion matrix at four weeks after transplanting with standard deviation in parentheses.

\begin{tabular}{|c|c|c|c|}
\hline Class Label & GR & GS & Accuracy \\
\hline GR & $24.3(2.3)$ & $5.7(2.3)$ & $81.0 \%$ \\
\hline GS & $5.6(2.1)$ & $21.4(2.1)$ & $79.3 \%$ \\
\hline Overall Accuracy & & & $80.2 \%$ \\
\hline
\end{tabular}


Table 4. Confusion matrix at five weeks after transplanting with standard deviation in parentheses.

\begin{tabular}{ccccc}
\hline & Class Label & GR & GS & Accuracy \\
\hline Actual Class & & & & \\
GR & $19.8(2.4)$ & $6.2(2.4)$ & $76.2 \%$ \\
GS & $5.9(2.3)$ & $17.1(2.3)$ & $74.4 \%$ \\
Overall Accuracy & & & $75.4 \%$ \\
\hline
\end{tabular}

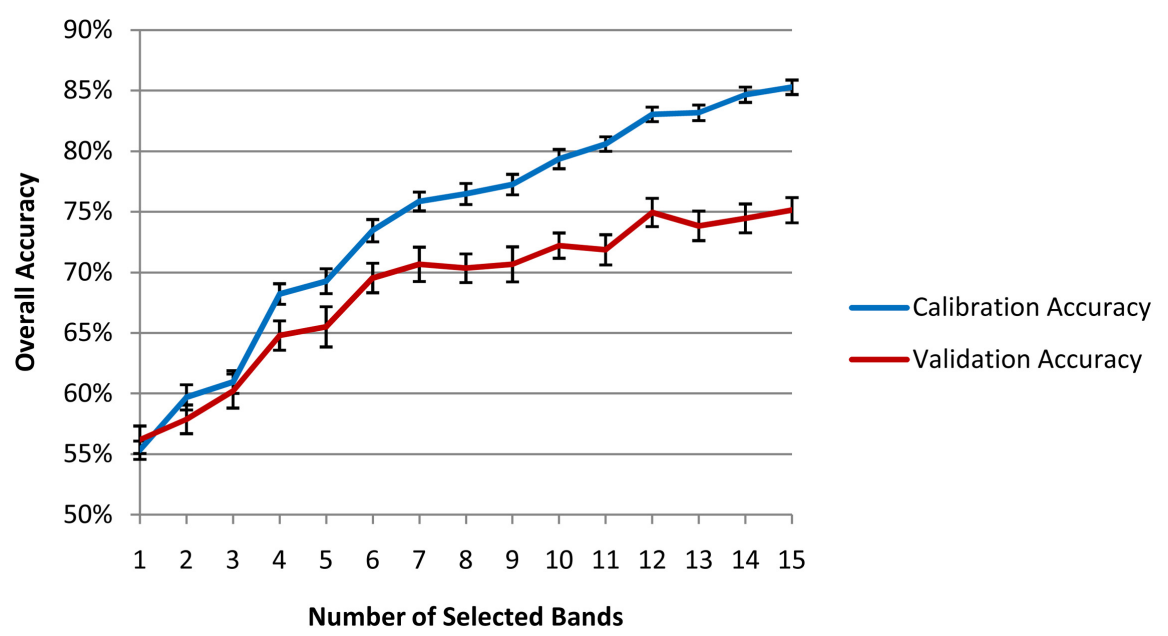

Figure 2. Plot of overall classification accuracy at three weeks after Italian ryegrass plants are transplanted. The error bars indicate a 95\% confidence interval on the mean of 100 permutations.

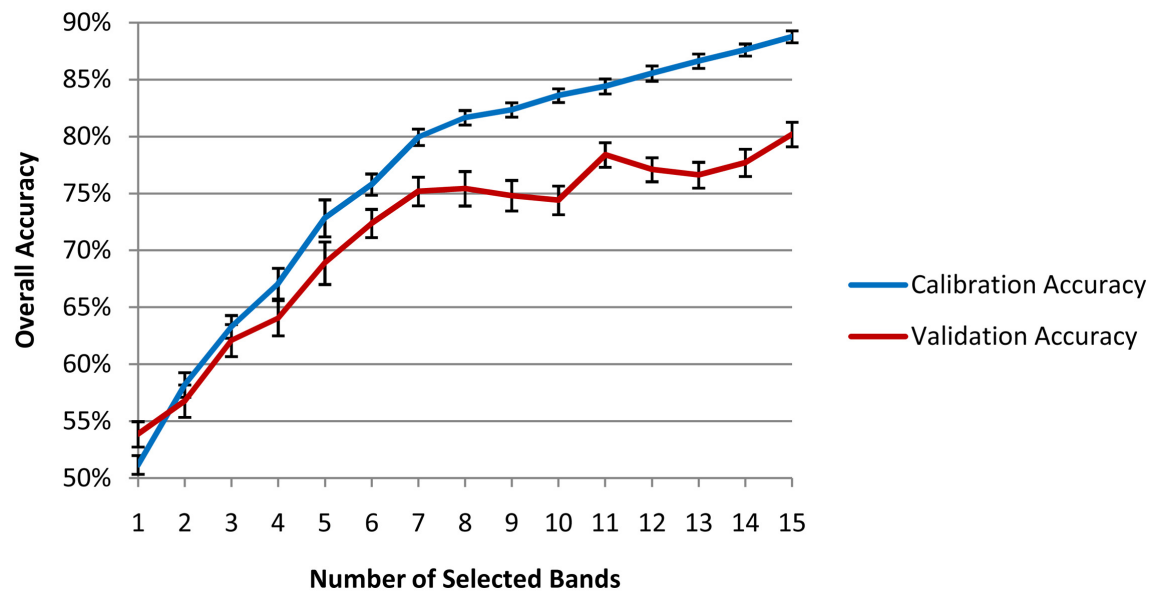

Figure 3. Plot of overall classification accuracy at four weeks after Italian ryegrass plants are transplanted. The error bars indicate a 95\% confidence interval on the mean of 100 permutations.

Our previous research on Palmer amaranth using similar data analysis techniques resulted in over $90 \%$ classification accuracy to differentiate GR and GS plants [9] while this research on Italian ryegrass has a classification accuracy in the range of $75 \%-80 \%$. Palmer amaranth is a summer annual broadleaf weed species and Italian ryegrass grows more vigorously in winter and early spring 


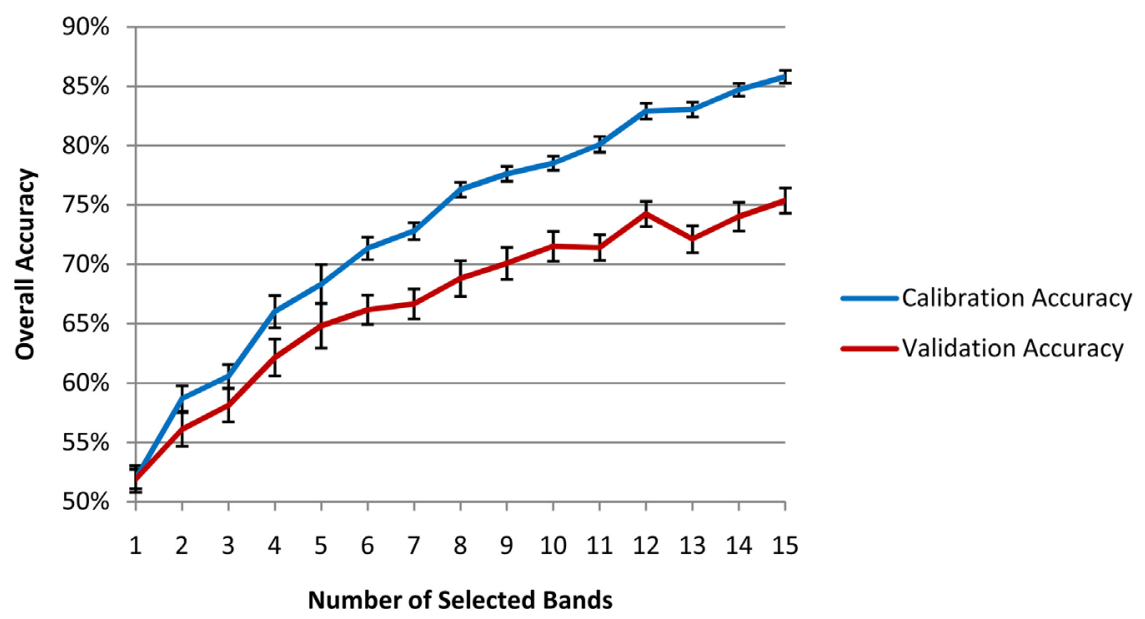

Figure 4. Plot of overall classification accuracy at five weeks after Italian ryegrass plants are transplanted. The error bars indicate a $95 \%$ confidence interval on the mean of 100 permutations.

with much narrower leaves. Although the performance for detection of Palmer amaranth and Italian ryegrass is different using hyperspectral imaging, the results of this study are still encouraging in illustrating that the approach developed for differentiating GR and GS Palmer amaranth could be transferred to Italian ryegrass with fairly high classification accuracy. In practice, a number of GR and GS weeds may coexist in a crop field, including Palmer amaranth and Italian ryegrass. In this case, it is possible to use hyperspectral imaging with similar data analysis techniques to detect GR Palmer amaranth and Italian ryegrass simultaneously with high success rate. For the time being, we are working on other GR weed species, such as Johnsongrass (Sorghum halepense), to see if the techniques with GR Palmer amaranth and Italian ryegrass could be further transferred. Furthermore, the analysis techniques used in this study might be limited to handle data complexity and nonlinearity. In this way more advanced methods will be evaluated and developed, such as machine learning and nonlinear analysis, when more training data are available with more experiments to produce better improved performance for detection of GR weeds for crop production management.

The difference in GR and GS classifications between images collected at three, four and five weeks after transplanting may indicate the difference in leaf epicuticular wax content between the different plant growth periods. A further research is needed to test the hypothesis. In addition plant imaging could be conducted at more time periods such as one or two weeks after transplanting at earlier growth stages and six or seven weeks after transplanting at later growth period for a broader comparison. Perhaps, less number of plants could be imaged at each growth period to balance the equipment and labor resources spent on imaging at more growth periods.

One drawback with the analysis used for the Italian ryegrass in this study was that the sensitive bands in each permutation varied because the training set was 
different with each permutation. In order to determine what the common sensitive bands are, a meta analysis is needed with more sample plants to be imaged and analyzed. With more data in such an approach, more accurate probability distributions of GR and GS plants could be estimated to more reliably determine the wavelength bands that are sensitive to be useful for analysis.

\section{Conclusion}

This study developed the methods of hyperspectral image analysis and data classification for GR and GS Italian ryegrass differentiation by adopting the methods we developed previously for GR and GS Palmer amaranth differentiation. The results indicated that the overall classification accuracy is between $75 \%$ and $80 \%$. Although the accuracy varies from the classification of Palmer amaranth in our previous study, this study proves that the success of the methods on one GR species can be transferred to another species and provides a rapid, non-destructive approach to differentiate between GR and GS Italian ryegrass for improved site-specific weed management.

\section{Disclaimer}

Mention of trade names or commercial products in this publication is solely for the purpose of providing specific information and does not imply recommendation or endorsement by the US Department of Agriculture.

\section{Acknowledgements}

The authors thank Jason Corbitt, Efren Ford, and Earl Gordon for technical assistance with growing the Italian ryegrass and assisting with data collection efforts.

\section{References}

[1] Duke, S.O., Lydon, J., Koskinen, W.C., Moorman, T.B., Chaney, R.L. and Hammerschmidt, R. (2012) Glyphosate Effects on Plant Mineral Nutrition, Crop Rhizophere Microbiota, and Plant Disease in Glyphosate-Resistant Crops. Journal of Agricultural and Food Chemistry, 60, 10375-10397. https://doi.org/10.1021/jf302436u

[2] Heap, I.M. (2018) International Survey of Herbicide Resistant Weeds. http://www.weedscience.org/

[3] Koger, C.H. and Reddy, K.N. (2005) Role of Absorption and Translocation in the Mechanism of Glyphosate Resistance in Horseweed (Conyza canadensis). Weed Science, 53, 84-89. https://doi.org/10.1614/WS-04-102R

[4] Shaner, D.L., Nadler-Hassar, T., Henry, W.B. and Koger, C.H. (2005) A Rapid in Vivo Shikimate Accumulation Assay with Excised Leaf Discs. Weed Science, 53, 769-774. https://doi.org/10.1614/WS-05-009R.1

[5] Silva, F.B., Costa, A.C., Alves, R.R.P. and Megguer, C.A. (2014) Chlorophyll Fluorescence as an Indicator of Cellular Damage by Glyphosate Herbicide in Raphanus sativus L. Plants. American Journal of Plant Sciences, 5, 2509-2519. https://doi.org/10.4236/ajps.2014.516265 
[6] Mahlein, A.K., Steiner, U., Hillnhutter, C., Dehne, H.W. and Oerke, E.C. (2012) Hyperspectral Imaging for Small-Scale Analysis of Symptoms Caused by Different Sugar Beet Diseases. Plant Methods, 8, 3. https://doi.org/10.1186/1746-4811-8-3

[7] Ashourloo, D., Mobasheri, M. and Huete, A. (2014) Developing Two Spectral Disease Indices for Detection of Wheat Leaf Rust (Puccinia triticina). Remote Sensing, 6, 4723-4740. https://doi.org/10.3390/rs6064723

[8] Lowe, A., Harrison, N. and French, A.P. (2017) Hyperspectral Image Analysis Techniques for the Detection and Classification of the Early Onset of Plant Disease and Stress. Plant Methods, 13, 80. https://doi.org/10.1186/s13007-017-0233-Z

[9] Reddy, K.N., Huang, Y., Lee, M.A., Nandula, V.K., Fletcher, R.S., Thomson, S.J. and Zhao, F. (2014) Glyphosate-Resistant and -Susceptible Palmer Amaranth (Amaranthus palmeri S. Wats.): Hyperspectral Reflectance Properties of Plants and Potential for Classification. Pest Management Science, 70, 1910-1917. https://doi.org/10.1002/ps.3755

[10] Montgomery, D.C. and Runger, G.C. (2003) Applied Statistics and Probability for Engineers. 3rd Edition, John Wiley \& Sons, Inc., New York.

[11] Hughes, G. (1968) On the Mean Accuracy of Statistical Pattern Recognizers. IEEE Transactions on Information Theory, 14, 55-63. https://doi.org/10.1109/TIT.1968.1054102

[12] Green, D.M. and Swets, J.A. (1966) Signal Detection Theory and Psychophysics. John Wiley \& Sons, Inc., New York.

[13] Duda, R.O., Hart, P.E. and Stork, D.G. (2001) Pattern Classification. 2nd Edition, John Wiley \& Sons, Inc., New York. 